\title{
Relationship among resonant frequencies of Sierpinski multiband fractal antennas
}

\author{
Ivan R. Gonzalez-Rangel ${ }^{1}$, Genaro Hernandez-Valdez ${ }^{1, *}$, Edgar A. Andrade-Gonzalez ${ }^{1}$, Mario Reyes-Ayala ${ }^{1}$, J.R. \\ Miranda-Tello ${ }^{1}$, Jose Serrano-Chavez ${ }^{1}$ \\ ${ }^{1}$ Electronics Department, UAM-A, Mexico City
}

\begin{abstract}
In this paper, the relationships between the different resonance frequencies of Sierpinski fractal antennas of four-iterations are studied. In particular, Sierpinski fractal antennas with operating frequencies of the initial triangle of $250 \mathrm{MHz}, 350 \mathrm{MHz}$ and $530 \mathrm{MHz}$ were designed and built. The antennas are made of copper tablets with bakelite substrate. The performance of the designed antennas is measured in terms of return losses. The return losses are obtained experimentally with a "RFX" system that measures antenna parameters in conjunction with a network analyzer. These results are compared with numerical simulations of commercial finite-element program that analyzes high frequency electromagnetic structures "HFSS". Experimental and simulation results show that there is approximately a factor of 2 between the resonance frequencies of the first and second iterations and the second and third iterations.
\end{abstract}

Keywords: Fractal, multiband, Sierpinski

\section{Introduction}

For the design of antennas, fractal antennas are important to solve challenges related with lower-cost, multi-band applications, broadband, and miniaturization [1], [12]. The operation parameters of a fractal antenna strongly depend on the type of fractal geometry and materials used in its construction [1], [10-13].

Today, with the miniaturization of wireless devices there is an increased demand for smart antennas. The designs of these antennas don't have an easy way to get operating requirements. This is a challenge because the operating characteristics and performance of the antennas are affected significantly by their small sizes. Furthermore, operating in more than one frequency band is also a challenge in the design of the antennas [5-10].

Fractal antennas use fractal geometry in their design; these forms allow that a single antenna has the ability to simultaneously resonate at more than one frequency band. Fractal geometry maximizes the perimeter of the antenna in a given area. This reduces the size of the area of an antenna maintaining the same perimeter. The constructions of some fractal shapes have properties that allow them to have a multiband behaviour. Some ideals fractal shapes are self-similar. A classic example of selfsimilar fractal geometry is the Sierpinski triangle [3-6].

In this research, the methodology proposed in [5] is used to properly build monopole-type multiband fractal antennas using Sierpinski fractal geometry with four iterations. For building these antennas, copper is used for the active antenna element, bakelite as substrate, and
SMA type feeder. The contribution of this work is the study of the relationship between the resonance frequencies of the various iterations of the Sierpinski fractal antenna. The performance of the antennas studied is measured in terms of return loss. The return loss is measured experimentally using a measuring system parameter antenna in conjunction with a network analyzer. These results are compared with those obtained by finite element simulation; specifically, the High Frequency Electromagnetic Structures simulation package is used to this end (HFSS).

\section{Sierpinski Geometry}

The construction of the Sierpinski triangle is based on an initial equilateral triangle that represents the zero-th iteration. In the first iteration, it is drawn another equilateral triangle that is rotated 180 geometrical degrees, whose sides have a length equal to 0.5 times the length of the sides of the initial triangle. This triangle is suppressed from the initial triangle; the result of this procedure is shown in the second geometric shape of Fig. 1. So, in the first iteration there are three equilateral triangles inside the triangle of the zero-th iteration. If in each of these triangles the above process is repeated, the second Sierpinski iteration is obtained, this case is shown in the third geometric shape of Fig. 1. This process is repeated $N$ times according to the total number of desired iterations. The last geometric shape of Fig. 1 represents the Sierpinski triangle with $N=4$ iterations.

\footnotetext{
* Corresponding author: ghv@,correo.azc.uam.mx
} 


\subsection{Sierpinski triangle area}

After each transformation of the Sierpinski triangle, a fragment of the initial triangle is removed. The area of the triangle decreases three quarters of the initial area in each iteration, that is, the resulting area decreases by a factor $3 / 4$. Equation (1) calculates the area resulting from the $n$-th iteration.

$$
A_{\mathrm{n}}=A_{0}(3 / 4)^{n}
$$

where $A_{0}$ is the area of the initial triangle and $n$ is the iterations number. It is noted that increasing the number of iterations, the triangle area decreases, therefore, with a large number of iterations the area tends to zero [8].

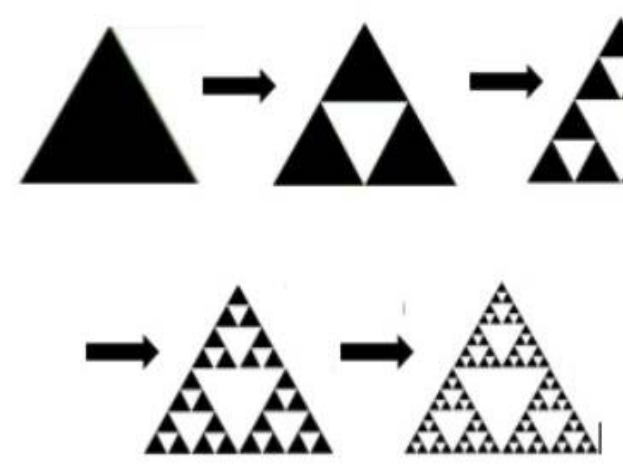

Fig. 1. Sierpinski triangle transformations for 4 iterations.

\subsection{Sierpinski triangle perimeter}

As discussed above, the initial triangle is transformed into three self-similar shapes after each iteration. Fig. 1 shows that each of the sides of the triangles of the current iteration has a length equal to 0.5 times the length of the sides of the triangles of the previous iteration. After deleting the triangles that are rotated 180 geometrical degrees, the perimeter of the resulting fractal figure can be calculated with equation (2).

$$
P_{n}=P_{0}(3 / 2)^{n}
$$

where $P_{0}$ is the initial perimeter of the triangle and $n$ is the iteration number. It is noted that as the number of iterations increases, the perimeter of the antenna tends to infinity [8].

\section{Sierpinski Monopole}

The Sierpinski monopole is a fractal antenna based on the Sierpinski triangle. The number of iterations is directly related to the number of frequency bands in which the antenna can operate. However, having a very large number of iterations results in both a significant decrease in the area of the antenna and considerable increase in its perimeter, such that the effectiveness of the antenna will be reduced [4-7]. This is why the number iterations should not be too large. The height of each one of the triangles that there are in the antenna corresponds to a frequency band. These heights are shown in Fig. 2.

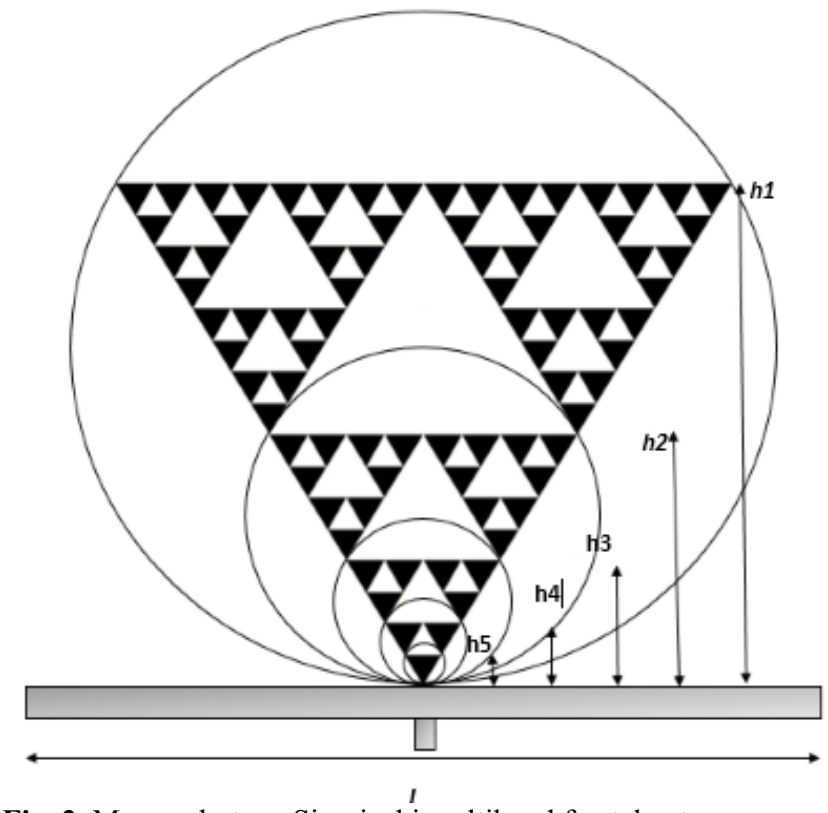

Fig. 2. Monopole-type Sierpinski multiband fractal antenna

The resonance frequency of the zero-th iteration of the antenna is calculated using (3).

$$
f \approx k \frac{c}{h}
$$

where $f$ is the resonance frequency of the first iteration of the antenna, $c$ is the value of the speed of light in vacuum, $h$ is the height value of the larger triangle of the antenna, and $k$ is a constant with value 0.142 . The factor $k$ is smaller than 1 , so this determines how small is a fractal antenna compared against a conventional antenna. This paper considers three Sierpinski antennas with resonant frequencies for the zero-th iteration equal to $250 \mathrm{MHz}, 350 \mathrm{MHz}$, and $530 \mathrm{MHz}$.

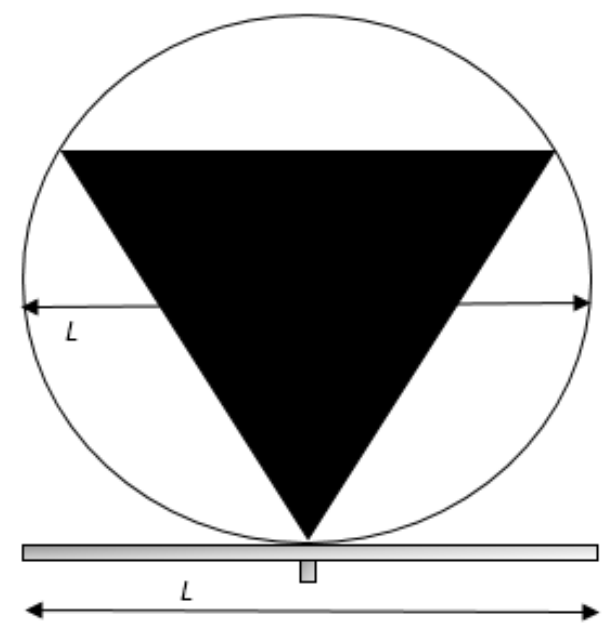

Fig. 3. Ground plane and the diameter of the zero-th iteration of the Sierpinski fractal antenna.

The Copper antennae are made over a bakelite plate with a relative permittivity $\varepsilon_{\mathrm{r}}=4.8$ and thickness of $1.565 \mathrm{~mm}$. In the resulting triangle, after the first 
iteration, it is considered a circle with diameter $L$ which touches its vertices, as it is illustrated in Fig. 3. The ground plane is on the opposite side of the bakelite plate and has an area equal to $L \cdot L$, according to the frequency of each of the three developed antennas.

\section{Simulation Results}

We used HFSS (High Frequency Structural Simulator) software of ANSYS, which is a simulator for structures and devices that respond at high frequencies. This program maintains high standards of precision; it performs an analytical solution that is based on finite element integral equations and hybrid methods for solving a wide range of microwave, radio frequency and digital high speed applications.

The HFSS simulator offers a wide range of materials, so it is important to choose the correct type of material used in each structure in order that the simulation results are closer to the experimental results.

The antenna design within the simulator comprises a part of copper, which is the antenna structure with a thickness of $0.035 \mathrm{~mm}$; a substrate that simulates the printed circuit board (a substrate of bakelite was chosen with a thickness of $1.565 \mathrm{~mm}$ ). The simulator uses the default parameters of this material, one of these parameters is the relative permittivity $\left(\varepsilon_{\mathrm{r}}=4.8\right)$. Copper was chosen for the ground plane, and an SMA connector was chosen as a feeder.

The frequency sweep was adjusted to the range from $10 \mathrm{MHz}$ to $8 \mathrm{GHz}$. The simulator has three types of frequency sweeps, the first one uses the discrete Fourier transform, the second method uses the fast Fourier transform, and the last method uses interpolation. For this work, the method of the fast Fourier transform was used. An option was also included to normalize ports with an impedance of $50 \mathrm{ohms}$.

The simulations were performed with a resolution of $10 \mathrm{MHz}$ to obtain the resonant frequencies of the antennas with a high level of accuracy. In Fig 4, the simulated structure in HFSS is presented.

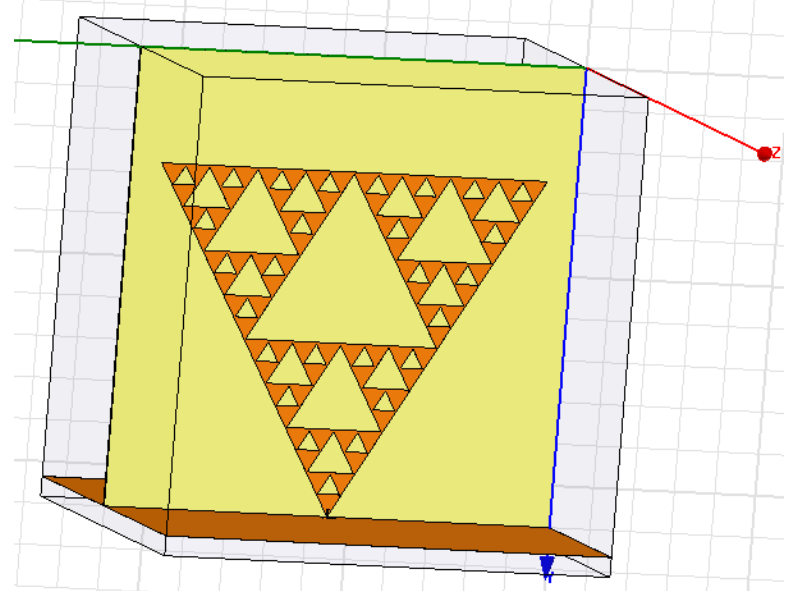

Fig. 4. Simulated structure in HFSS.

Simulation results for antennas Sierpinski with maximum height of $8 \mathrm{~cm}, 12 \mathrm{~cm}$, and $16.5 \mathrm{~cm}$ are shown in Figs. 5, 6, and 7, respectively. These heights correspond to the frequencies of the zero-th iteration equal to $530 \mathrm{MHz}, 350 \mathrm{MHz}$, and $250 \mathrm{MHz}$, respectively.

Authors in [5] state that, if both the contribution of the center triangle shaped holes to the antenna performance in each iteration are neglected (white triangle shaped regions in Fig. 2) and it is assumed that the current flowing from the feeder is concentrated over a region that is comparable in size to the wavelength, a behavior similar to five-scaled bowtie antenna (each one operating at its resonant frequency) is expected. Due to the fact that the scale factor among the five heights shown in Fig. 2 is 2, authors in [5] concluded that a similar behavior between the resonant frequencies of the Sierpinski multiband antenna is expected. Figs. 5-7 shows that the bands in which the Sierpinski antenna resonates in the $n$-th iteration are adjusted by a certain factor to the resonant frequency of the $(n+1)$-th iteration [4-7]. For instance, Fig. 6 shows that between the resonant frequencies of the following iterations: zero-th and first, first and second, second and third, and third and fourth, there exist an approximate factor of 3.1, 1.8, 1.68 , and 1.47 , respectively. These results differ from the expected value of 2 that is justified in [5]. In the next section, our simulated results are experimentally verified.

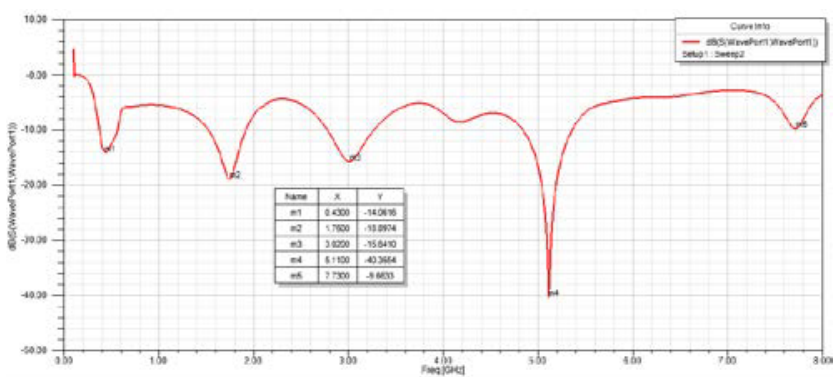

Fig. 5. Return loss of the antenna of $8 \mathrm{~cm}(530 \mathrm{MHz})$.

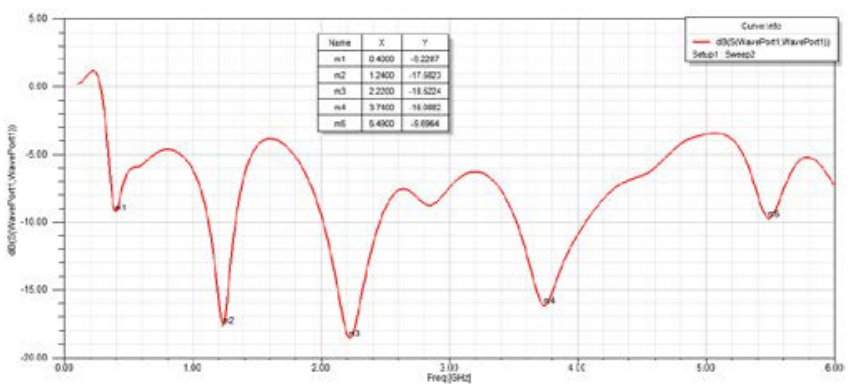

Fig. 6. Return loss for the antenna of $12 \mathrm{~cm}(350 \mathrm{MHz})$.

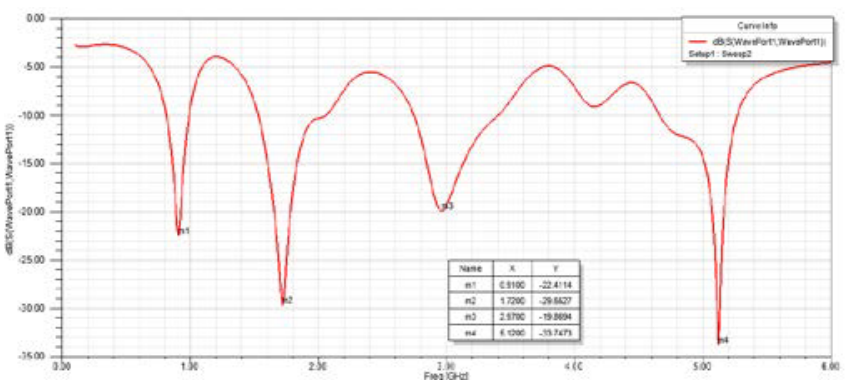

Fig. 7. Return loss for the antenna of $16.5 \mathrm{~cm}(250 \mathrm{MHz})$. 


\section{Experimental Results.}

This section presents the experimental results of the return losses of Sierpinski antennas designed and simulated in previous sections. These antennas are experimentally analyzed using a measure device of antenna pattern, which measures the electromagnetic field in two orthogonal directions within the near field of the antenna under study. These measurements are used to estimate the far field distribution using flat opening for transforming the angular spectrum or spectral transformation plane wave (PSW). Then a second custom algorithm adjusts the projection of the far field to remove predictable coupling effects in measurements. This algorithm also takes into account the compensation response. This measurement system is capable of operating from $300 \mathrm{MHz}$ to $6 \mathrm{GHz}$ with a resolution of $10 \mathrm{MHz}$. The results obtained are shown in Figs. 8-10.

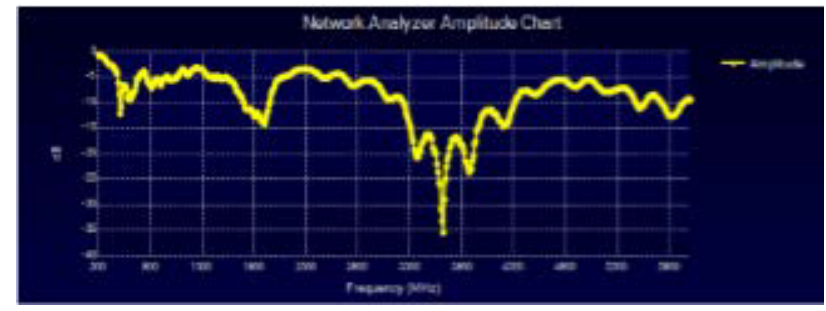

Fig. 8. Experimental measure of the return loss in the antenna of $8 \mathrm{~cm}(530 \mathrm{MHz})$.

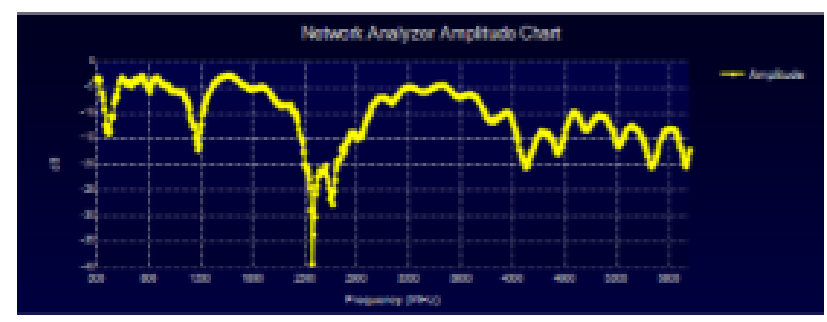

Fig. 9. Experimental measure of the return loss in the antenna of $12 \mathrm{~cm}(350 \mathrm{MHz})$.

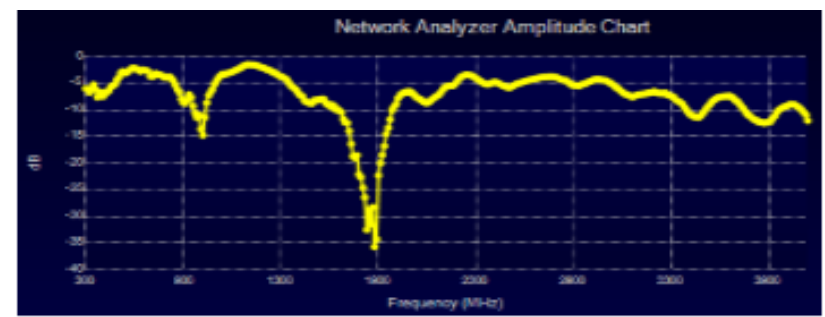

Fig. 10. Experimental measure of the return loss in the antenna of $16 \mathrm{~cm}(250 \mathrm{MHz})$.

In the fifth column of Tables I, II, and III is shown the factor between the resonance frequencies of two adjacent iterations. In particular, from the values reported in this column, it is observed that, regardless of the designed resonant frequency of iteration zero, the factor between the resonance frequency of the first and second iteration is close to two. Note that, in general, this factor depends on the iteration number, resonant frequency of the iteration zero, longitude and permittivity constant of the substrate.
Figs. 8, 9, and 10 show the return loss obtained experimentally for antennas with a maximum height of 8 $\mathrm{cm}, 12 \mathrm{~cm}$, and $16 \mathrm{~cm}$, respectively. The experimental and simulated results presented in Figs. 5-10 are summarized in Tables I, II, and III. Tables I, II y III, correspond to the Sierpinski fractal antenna with a maximum height of $8 \mathrm{~cm}, 12 \mathrm{~cm}$ and $16.5 \mathrm{~cm}$, respectively. In the third column of Tables I, II y III, the simulated resonant frequencies of the different iterations of the fractal Sierpinski antennas are shown. On the other hand, in the fourth column of Tables I, II and III the experimental results of the resonance frequencies of the different iterations of Sierpinski antennae under study are shown. In these tables a high correlation between experimental and simulation results is observed; this is especially true for the second and third iteration.

Table 1. Sierpinski fractal antenna of $8 \mathrm{~cm}(530 \mathrm{MHz})$.

\begin{tabular}{|c|c|c|c|c|}
\hline Iteration & $\begin{array}{c}\text { Height } \\
(\mathbf{c m})\end{array}$ & $\begin{array}{c}\text { Simulated } \\
\text { frequency } \\
(\mathbf{G H z})\end{array}$ & $\begin{array}{c}\text { Experimental } \\
\text { frequency } \\
(\mathbf{G H z})\end{array}$ & $\begin{array}{c}\text { Factor } \\
\boldsymbol{f}_{\boldsymbol{n}+\mathbf{1}} / \boldsymbol{f}_{\boldsymbol{n}}\end{array}$ \\
\hline 0 & 8 & 0.43 & 0.51 & 3.72 \\
\hline 1 & 4 & 1.75 & 1.90 & 1.91 \\
\hline 2 & 2 & 3.02 & 3.62 & 1.52 \\
\hline 3 & 1 & 5.11 & 5.52 & 1.05 \\
\hline 4 & 0.5 & 7.7 & 5.82 & --- \\
\hline
\end{tabular}

Table 2. Sierpinski fractal antenna of $12 \mathrm{~cm}(350 \mathrm{MHz})$.

\begin{tabular}{|c|c|c|c|c|}
\hline Iteration & $\begin{array}{c}\text { Height } \\
(\mathbf{c m})\end{array}$ & $\begin{array}{c}\text { Simulated } \\
\text { frequency } \\
(\mathbf{G H z})\end{array}$ & $\begin{array}{c}\text { Experimental } \\
\text { frequency } \\
(\mathbf{G H z})\end{array}$ & $\begin{array}{c}\text { Factor } \\
\boldsymbol{f}_{\boldsymbol{n} \mathbf{1}} / \boldsymbol{f}_{\boldsymbol{n}}\end{array}$ \\
\hline 0 & 12 & 0.40 & 0.41 & 3.10 \\
\hline 1 & 6 & 1.24 & 1.27 & 1.87 \\
\hline 2 & 3 & 2.23 & 2.37 & 1.87 \\
\hline 3 & 1.5 & 3.74 & 4.44 & 1.27 \\
\hline 4 & 0.75 & 5.50 & 5.64 & --- \\
\hline
\end{tabular}

Table 3. Sierpinski fractal antenna of $16.5 \mathrm{~cm}(250 \mathrm{MHz})$

\begin{tabular}{|c|c|c|c|c|}
\hline Iteración & $\begin{array}{c}\text { Altura } \\
(\mathbf{c m})\end{array}$ & $\begin{array}{c}\text { Frecuencia } \\
\text { simulada } \\
(\mathbf{G H z})\end{array}$ & $\begin{array}{c}\text { Frecuencia } \\
\text { experimental } \\
(\mathbf{G H z})\end{array}$ & $\begin{array}{c}\text { Factor } \\
\boldsymbol{f}_{\boldsymbol{n}+\mathbf{1}} / \boldsymbol{f}_{\boldsymbol{n}}\end{array}$ \\
\hline 0 & 16.5 & --- & --- & --- \\
\hline 1 & 8.25 & 0.91 & 0.90 & 2 \\
\hline 2 & 4.12 & 1.72 & 1.78 & 1.92 \\
\hline 3 & 2.06 & 2.97 & 3.42 & 1.87 \\
\hline 4 & 1.03 & 5.12 & 3.78 & 1.11 \\
\hline
\end{tabular}

\section{Conclusions}

The ratios between the resonance frequencies of the various iterations of the fractal Sierpinski multiband antenna were investigated. To this end, three Sierpinski antennas of four iterations with operating frequencies in the iteration zero of 250,350 and $530 \mathrm{MHz}$ were designed, simulated, and built. The results show that only between the first to second, and second to third iterations there is a factor around two. For any other pair of adjacent iterations, the factor between the resonance 
frequencies depends on the designed value of the frequency of the iteration zero and other design parameters of the antenna (thickness and dielectric constant of the substrate, etc.)

\section{References}

1. Balanais Constantine A. Modern antenna handbook (Wiley. Canada, 2008).

2. Andreas F. Molisch. Wireless Communications (IEEE PRESS, 2006).

3. Cardama Aznar Ángel. Antenas (Alfaomega, 2004)

4. Muhammad Waqas. "Multiband Sierpinski Fractal Antennas". IEEE 13th International Multitopic Conference, 2009. INMIC 2009. 1-6. (Dec 2009).

5. C. Puente, J. Romeu, R. Pous and A. Cardama, "On the Behavior of the Sierpinski Multiband Fractal Antenna". IEEE Transactions on Antennas and Propagation, Vol. 46, No. 4, (Abril 1998).

6. MReza Khorramian, Bijan Zakeri, Ata Ebrahimzadeh, "Design and Analysis of a Multiband Sierpinski Type Monopole Fractal Antenna". IEEE Conference Publications. 21st Telecomunications Forum, 675-677, (2013).

7. C. Puente, J. Romeu; R. Pous, X. Garcia, F. Benitez. "Fractal Multiband Antenna Based on the Sierpinski Gasket”. IET Journals \& Magazines Electronics Letters, vol. 32, 1-2. (1996).

8. González R. Ivan R. "Antena fractal de Sierpinski para operar en las bandas de $900 \mathrm{MHz}$ y $1.8 \mathrm{GHz}$," Pistas Educativas No. 120,143-159, (October 2016).

9. Naresh Kumar, N.; Saran Krishna, K.; Choukiker, Y.K.; Behera, S.K., "Compact modified Sierpinski fractal monopole antenna for multiband wireless applications," IEEE Conference Publications, 12641266. (2014).

10. Yuan-hai Yu; Chang-peng Ji., "Research of fractal technology in the design of multi-frequency antenna," IEEE Conference Publications, 1-4. (2011).

11. Nayak, P.B.; Verma, S.; Kumar, P., "Multiband fractal antenna design for Cognitive radio applications," IEEE Conference Publications, 115120. (2013).

12. Werner, D.H.; Ganguly, S., "An overview of fractal antenna engineering research," IEEE Journals \& Magazines, 38-57. (2003).

13. Dwivedi, R.P.; Upadhyay, D., "High gain dual band antenna using fractal geometry for mobile communication," IEEE Conference Publications, 50-55. (2015). 